\title{
Complicaciones intraoperatorias del mapeo corticosubcortical
}

\author{
P. Teixidor; R. García; M. Alamar; M. González*; R. Llasera*; M.J. Durá**; J. Muñoz y R. Florensa
}

Servicio de Neurocirugía. Servicio de Anestesia*. Servicio de Rehabilitación**. Hospital Germans Trias y Pujol. Badalona. Barcelona.

Resumen

Mediante una revisión de nuestra casuística y de la literatura analizamos las complicaciones intraoperatorias derivadas del uso de las estimulaciones cerebrales directas bajo anestesia general y anestesia local y sedación. Se realizó un estudio retrospectivo descriptivo de los pacientes intervenidos (2004-2008) en que se utilizó la técnica del mapeo corticosubcortical. Se determinaron como variables preoperatorias comunes: edad, sexo, localización tumoral, anatomía patológica, y en los pacientes operados despiertos, se registró el ASA, IMC y duración de la intervención. En los dos grupos se constataron la aparición de edema cerebral o crisis comiciales. En los pacientes despiertos se registró además las complicaciones respiratorias, hemodinámicas, toxicidad por anestésico local, nivel inadecuado de sedación, náuseas/vómitos, dolor e incomodidad postural. Bajo anestesia local y sedación se operaron 10, de 41 años de edad media, IMC medio de 26,8 y un ASA preoperatorio de I o II . La duración media de la intervención fue de 5 horas y 20 minutos. Mediante anestesia general se intervinieron 10 pacientes, de 55 años de media. En ningún caso de los dos grupos se constató edema cerebral, 4 pacientes presentaron crisis comiciales (autolimitadas con irrigación de suero frío). En 5 pacientes con anestesia local y sedación no registramos ninguna complicación, 2 pacientes episodios de desaturación sin complicaciones, 3 episodios de hipertensión y 2 incomodidad postural. Ninguna de las complicaciones intraoperatorias que registramos interfirió en el desarrollo de la cirugía. Es una técnica segura si se realiza siguiendo las indicaciones y los parámetros recomendados. Las crisis y complicaciones respiratorias son las complicaciones más frecuentes y a su vez las más temidas, puesto que pueden influir en casos problemáticos en el proceso de la cirugía.

PALABRAS CLAVE: Mapeo cerebral. Complicaciones

Recibido: 2-02-09. Aceptado: 23-09-09 intraoperatorias. Estimulaciones cerebrales directas.

Intraoperative complications of corticosubcortical mapping

Summary

By looking through our cases and literature, an analysis of the surgical complications derived from direct cerebral stimulation under general anesthesia and local anesthesia and sedation was made. A retrospective descriptive study was performed including patients who were intervened in our centre from 2004 to 2008 and had the cortico-subcortical mapping technique. Common pre-operation variables were as follow: Age, sex, tumor localization and tumor's pathology; On patients intervened while awake, we collected the ASA, BMI and duration of the intervention. Afterwards, variable like epileptic attacks and cerebral edema were included in two groups. In addition, on those awake, respiratory and circulatory complications, local anesthesia toxicity, poor level of sedation, nausea and vomiting, pain and feeling uncomfortable with body posture were collected as well. A total of 20 patients had surgery. 10 of them were operated under local anesthesia and sedation with a mean age of 41 years, mean BMI of 26.8 and a pre-operatory ASA score of I or II ( except one patient with ASA III). The mean time duration of the surgical procedure was 5 hours and 20 minutes. On the other side, 10 patients were intervened with general anesthesia with a mean age of 55 years. There were no cases of cerebral edema in either group, although in 4 patients had epileptic attacks which resolved with cold saline irrigation. Five patients with local anesthesia and sedation did not have any complication, 2 patients showed desaturation episodes without further complications, three manifested hypertension episodes and

Abreviaturas. ASA: Clasificación del grado de riesgo anestésico de la Sociedad Americana de Anestesia. ICM: Índice de masa corporal. 
two signs of feeling uncomfortable with body posture. None of the intra-operatory complications registered interfered wit the overall surgical procedure. This is a safe technique if performed following recommended indications and parameters. Regarding complications, respiratory complications are the most common and the most dangerous, as they can influence in the surgical procedure for difficult cases.

KEY WORDS: Brain mapping. Intraoperative complications. Direct cerebral stimulation.

\section{Introducción}

El uso de la técnica de la estimulación cerebral directa en neurocirugía, nos aporta básicamente dos aspectos. En primer lugar, conlleva una optimización de la ratio beneficio/riesgo de la cirugía en áreas elocuentes. Permite maximizar el grado de resección tumoral y a su vez, disminuye las secuelas definitivas (menos del 5\% de los casos), hecho que genera un aumento de la calidad de vida en estos pacientes. En segundo lugar, proporciona la integración de nuevos conceptos en la estrategia quirúrgica, ampliando el conocimiento de la organización funcional cerebral (tanto a nivel cortical como subcortical) y de la plasticidad cerebral $^{4,6,7}$.

Los pioneros de la estimulación cortical fueron Cushing en 1909 y posteriormente Penfield (1937) y Forester (1939), mientras que la estimulación subcortical fue descrita inicialmente por Berger en el año $1990^{2}$. En España, una de las primeras cirugías en que se utilizó mapeo cortical fue practicada por el Dr. Conesa en el año 1989.

Aún con los avances de las técnicas de diagnóstico por imagen y neuronavegación que conllevan una localización intraoperatoria cada vez más precisa de las lesiones tumorales respecto la organización funcional, todavía no podemos sustituir el mapeo intraoperatorio.

Con los años se ha ido difundiendo su utilización en los quirófanos neuroquirúrgicos puesto que se ha demostrado que las estimulaciones cerebrales directas son fiables, reproducibles, no lesivas y precisas ${ }^{5}$. Su práctica en neurooncología se emplea para una gran variedad de localizaciones tumorales así como diferentes tipos histológicos ${ }^{15}$. Cada vez más su uso, se ha extendido no sólo en la cirugía tumoral, si no además en la cirugía de la epilepsia, del dolor, etc...

Como cualquier técnica neuroquirúrgica, requiere de una curva de aprendizaje no sólo para el neurocirujano si no además para todo el equipo quirúrgico. Asimismo su práctica no está exenta de complicaciones. Estas complicaciones deben ser conocidas y previstas por todo el equipo puesto que una rápida actuación puede permitir un fácil manejo sin comportar riesgos para el paciente ni una variación en el procedimiento quirúrgico, en la mayor parte de los casos.

Mediante una revisión de nuestra casuística y de la literatura analizamos las complicaciones intraoperatorias derivadas del uso de las estimulaciones cerebrales directas bajo anestesia general y anestesia local y sedación.

\section{Material y métodos}

Realizamos un estudio retrospectivo observacional de los pacientes intervenidos en nuestro centro (Hospital Germans Trias i Pujol) entre el mes de noviembre del 2004 y enero del 2008. En todos ellos se utilizó las estimulaciones cerebrales directas para el mapeo motor mediante anestesia general o mapeo lenguaje y/o sensitivo/motor con anestesia local y sedación.

La técnica utilizada en todos ellos fue la técnica de cartografía presentada por Berger y modificada por Duffau ${ }^{5}$. El generador utilizado para las estimulaciones fue el generador de Ojemann. Se usó una sonda bipolar con contactos espaciados en $5 \mathrm{~mm}$. La frecuencia de pulso fue de $60 \mathrm{~Hz}$, con una duración de fase de $1 \mathrm{~ms}$, una amplitud de 1 a $6 \mathrm{~mA}$ bajo anestesia local y 2 y $18 \mathrm{~mA}$ bajo anestesia general.

Desde el punto de vista anestésico para los pacientes despiertos se siguió el protocolo habitual en nuestro centro para este tipo de cirugía según la técnica "dormido, despierto, dormido". Se utilizó la infusión de propofol y remifentanilo en modo TCI (TCI: target controlled infusion), parándose cuando se debía despertar al paciente para su colaboración en el mapeo. Durante la inducción se pautó profilaxis antiemética mediante dexametasona $4 \mathrm{mg}$ y ondansetrón $4 \mathrm{mg}$. Todos los pacientes mantuvieron la ventilación espontánea durante todo el procedimiento, con aporte suplementario de oxígeno mediante cánula nasal de $\mathrm{O}_{2}$, y sin necesidad de otros dispositivos laríngeos. A todos los pacientes en el momento de la apertura dural se administró manitol al 20\% $0.5-1 \mathrm{~g} / \mathrm{Kg}$, y todos (salvo contraindicaciones) recibieron como analgesia: paracetamol $1 \mathrm{gr}$ endovenoso, metamizol $2 \mathrm{~g}$ endovenoso, y morfina 0.1 $0.15 \mathrm{mg} / \mathrm{Kg}$ endovenosa al acabar la cirugía.

Como monitorización intraoperatoria rutinaria se empleó el ECG, la presión sanguínea invasiva, la cánula nasal con analizador de $\mathrm{CO}_{2}$, el BIS, la sonda urinaria y control de diuresis y los gases arteriales sanguíneos.

Una vez inducida la anestesia, aseguramos la analgesia mediante la infiltración de anestésico local. Por protocolo, habitualmente utilizamos bupivacaína al $0,25 \%$ con adrenalina sin sobrepasar la dosis de $3 \mathrm{mg} / \mathrm{kg}$, que fue aplicada no sólo en la zona de la incisión si no que además se aplicó en las zonas que se prevea de retracción del cuero cabelludo durante la cirugía. También fue infiltrado el músculo temporal u occipital según el abordaje. Antes de abrir la duramadre, la infiltramos siempre con lidocaína $2 \% \sin$ 
Tabla 1

Resultados de los pacientes intervenidos bajo anestesia local y sedación

\begin{tabular}{|c|c|c|c|c|c|c|c|c|c|}
\hline Año & Sexo & Edad & $\begin{array}{c}\text { KPS } \\
\text { PREQ }\end{array}$ & Localización & $\begin{array}{l}\text { Anatomía } \\
\text { patológica }\end{array}$ & IMC & ASA & IQ (h) & Complicaciones \\
\hline 2005 & q & 32 & 90 & $\begin{array}{l}\text { Temporal } \\
\text { izquierdo }\end{array}$ & $\begin{array}{l}\text { Astrocitoma } \\
\text { grado III }\end{array}$ & 23.4 & II & 6.30 & $\begin{array}{l}\text { Incomodidad } \\
\text { postural/HTA }\end{array}$ \\
\hline 2005 & q & 60 & 90 & $\begin{array}{l}\text { Parietal } \\
\text { superior iz. }\end{array}$ & $\begin{array}{l}\text { Astrocitoma } \\
\text { grado III }\end{array}$ & 28 & II & 6.15 & $\begin{array}{l}\text { Incomodidad } \\
\text { postural/HTA/ } \\
\text { desaturación }\end{array}$ \\
\hline 2005 & $\hat{\sigma}$ & 63 & 100 & Temporal iz. & ODG grado III & 23.4 & II & 5.3 & $\bullet$ \\
\hline 2007 & q & 35 & 90 & $\begin{array}{l}\text { Frontotemporal } \\
\text { iz. }\end{array}$ & ODG grado II & 44.5 & II & 6 & Crisis comicial \\
\hline 2007 & $0^{\lambda}$ & 31 & 90 & $\begin{array}{l}\text { Frontoparietal } \\
\text { iz. }\end{array}$ & $\begin{array}{l}\text { Astrocitoma } \\
\text { grado II }\end{array}$ & 23.1 & I & 4.30 & $\begin{array}{l}\text { Episodio } \\
\text { desaturación/ } \\
\text { episodios } \\
\text { HTA/crisis } \\
\text { comicial }\end{array}$ \\
\hline 2007 & $0^{\pi}$ & 18 & 90 & Temporal d. & Cavernoma & 20.5 & I & 5 & $\bullet$ \\
\hline 2007 & q & 20 & 100 & Parietal iz. & $\begin{array}{l}\text { Astrocitoma } \\
\text { pilocítico }\end{array}$ & 22 & I & 5.20 & Episodio HTA \\
\hline 2007 & 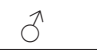 & 55 & 100 & Parietal iz. & Cavernoma & 37 & III & 5 & $\bullet$ \\
\hline 2007 & 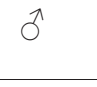 & 53 & 90 & Parietal iz. & $\begin{array}{l}\text { Astrocitoma } \\
\text { grado II }\end{array}$ & 24.2 & II & 5.30 & $\cdot$ \\
\hline 2008 & $q$ & 44 & 90 & Parietal iz. & ODG grado III & 22.4 & II & 5 & $\cdot$ \\
\hline Media & & 41 & 90 & & & 26.8 & II & 5.3 & \\
\hline
\end{tabular}

- Sin complicaciones. KPS: Karnofsky performance status.IMC: índice de masa corporal $\left(\mathrm{kg} / \mathrm{m}^{2}\right)$. ASA: American Society of Anesthesiologists. Clasificación según la impresión global de la complejidad médica del paciente (de I a V). IQ (h): intervención quirúrgica (horas). ODG: oligodendroglioma. HTA: hipertensión.

adrenalina (aproximadamente nunca más de $10 \mathrm{ml}$ ), con la ayuda de una aguja muy fina (tipo insulina), principalmente en la zona basal.

Para los pacientes dormidos se siguió el protocolo anestésico usual para una craneotomía habitual, salvo la utilización exclusiva de relajantes musculares de vida media corta para la intubación.

Las variables registradas fueron: las epidemiológicas (año de la intervención quirúrgica, edad y sexo del paciente), clínicas (KPS del paciente (Karnofsky performance status), localización y anatomía patológica de la lesión). En pacientes intervenidos despiertos: registramos el ASA (Clasificación según la impresión global de la complejidad médica del paciente de I a V, según la American Society of Anesthesiologists ), IMC (IMC: índice de masa corporal), y modo de colocación del paciente y duración de la intervención.

En todos los pacientes intervenidos registramos aquéllos que presentaron edema cerebral y crisis comiciales. En los pacientes despiertos estudiamos los que presentaron un exceso o nivel inadecuado de sedación, confusión, la percepción intraoperatoria de dolor, toxicidad por anestésico local, incomodidad postural, náuseas o vómitos, embolismo aéreo, obstrucción de vía aérea (apneas o hipercápneas), y complicaciones hemodinámicas (ya fuera hipertensión, hipotensión, taquicardia o bradicardia, o embolismo aéreo).

\section{Resultados}

Durante el período (noviembre 2004 y enero 2008) se intervinieron en nuestro centro un total de 20 pacientes, 10 bajo anestesia local y sedación y diez bajo anestesia general.

De los pacientes intervenidos despiertos, 5 fueron varones y 5 mujeres. La edad media era de 41 años con un rango entre 18 y 61 años. El índice de masa corporal medio fue de $26.8 \mathrm{~kg} / \mathrm{m}^{2}$, con un rango entre 20,5 y 44,5 (considerándose sobrepeso por encima de 25 , y por encima de 40 obesidad mórbida). El ASA preoperatorio fue de I o II en todos los pacientes salvo uno con ASA III. La duración media de la intervención fue de 5 horas y 30 minutos (ver tabla 1). Los tres primeros pacientes fueron colocados en decúbito late- 
Tabla 2

Resultados de los pacientes intervenidos bajo anestesia general

\begin{tabular}{|c|c|c|c|c|c|c|}
\hline Año & Sexo & Edad & KPS prequir & Localización & $\begin{array}{l}\text { Anatomía } \\
\text { patológica }\end{array}$ & $\begin{array}{l}\text { Complicaciones } \\
\text { intraoperatorias }\end{array}$ \\
\hline 2004 & $\sigma^{\pi}$ & 28 & 90 & Frontal izquierdo & Oligoastrocitoma grado III & Crisis comiciales \\
\hline 2005 & q & 54 & 90 & Frontal izquierdo & GBM & $\cdot$ \\
\hline 2005 & q & 74 & 80 & AMS derecha & GBM & $\bullet$ \\
\hline 2005 & $0^{\lambda}$ & 61 & 80 & Frontal izquierdo & GBM & • \\
\hline 2005 & q & 71 & 80 & Frontal izquierdo & GBM & $\bullet$ \\
\hline 2006 & q & 20 & 90 & Frontal derecho & Astrocitoma grado III & - \\
\hline 2006 & q & 75 & 80 & Frontal derecho & Metástasis & $\bullet$ \\
\hline 2006 & $0^{\pi}$ & 56 & 100 & Frontal derecho & MAV & $\bullet$ \\
\hline 2007 & 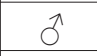 & 28 & 100 & Frontal izquierdo & Astrocitoma grado II & • \\
\hline 2008 & $0^{\pi}$ & 75 & 90 & Frontal derecho & Metástasis & Crisis comiciales \\
\hline Media & & 54,2 & 80 & & & \\
\hline
\end{tabular}

GBM: glioblastoma multiforme; MAV: malformación arteriovenosa. KPS: Karnofsky performance status. •: sin complicaciones

ral "completo". En los siguientes casos fueron colocados en decúbito supino parcialmente lateralizado, para conseguir un mayor confort del paciente durante la intervención.

El grupo de los diez pacientes intervenidos bajo anestesia general constó de 5 varones y 5 mujeres. La edad media fue de 55 años, con un rango entre 20 y 74 años (ver Tabla 2).

En ambos grupos no registramos ningún éxitus intraoperatorio, y ningún caso de edema cerebral.

Observamos cuatro casos de crisis comiciales, solventadas todas ellas con irrigación de suero frío. Dos casos se dieron en pacientes bajo anestesia general y dos casos bajo anestesia local y sedación.

En pacientes despiertos: 5 casos no padecieron ninguna complicación, 2 pacientes $(20 \%)$ presentaron episodios de desaturación que fueron resueltos sin complicaciones mediante ventilación con mascarilla durante unos instantes mientras se disminuía la sedación. Registramos en cuatro pacientes episodios de hipertensión arterial como complicaciones hemodinámicas. Mostraron una incomodidad postural importante dos pacientes, aunque no condicionó la finalización del mapeo. En ningún paciente se tuvo que proceder a una anestesia general. Tampoco se registró ningún paciente con signos de intoxicación por anestésico local, exceso o nivel inadecuado de sedación, confusión, náuseas o vómitos, dolor incontrolable que dificultara la continuidad del mapeo, o embolismo aéreo.

Ninguna de las complicaciones registradas interfirió en el desarrollo de la cirugía ni condicionó un cambio en la actitud quirúrgica planteada inicialmente.

\section{Discusión}

Los beneficios del empleo de la técnica de las estimulaciones cerebrales directas (corticales y subcorticales) son ampliamente conocidos y no son motivo de discusión en este estudio. A su vez, recordar que su uso no únicamente queda restringido en la neurooncología, sino que además puede ser utilizada en la cirugía de la epilepsia (destacar que los trabajos pioneros del mapeo cortical de Penfield y Ojemann fueron aplicados en cirugía de epilepsia), cirugía de lesiones vasculares (ejemplo malformaciones arteriovenosas...) o en la cirugía del dolor (por ejemplo para la colocación de electrodos corticales cerebrales...). Por el contrario, creemos que el conocimiento de las complicaciones que pueden surgir durante su utilización no ha sido muy tratado en la literatura. La obtención de una respuesta rápida y acertada frente a una complicación puede evitar riesgos para el paciente y tener que realizar modificaciones en el planteamiento de la cirugía.

Las complicaciones derivadas del uso de esta técnica comunes tanto en pacientes despiertos como en pacientes dormidos serían básicamente el edema cerebral, las crisis comiciales o la carencia de respuesta en el mapeo. Aunque la complicación sea similar, no podemos olvidar que el manejo de estas incidencias puede ser más dificultoso en un paciente bajo anestesia local y sedación.

Para todo tipo de cirugía es imprescindible una interacción entre anestesistas y cirujanos, pero ésta es mucho más destacada cuando el paciente se somete a una anestesia local y sedación para la obtención de unos buenos resultados. En cada momento de la cirugía, las demandas del 
neurocirujano respecto al grado de sedación del paciente pueden variar desde la sedación profunda hasta su perfecta colaboración. Una coordinación escrupulosa entre cirujanos y anestesistas en cada tiempo quirúrgico se hace del todo necesaria para un buen desenlace de la cirugía. De igual forma, la cirugía despierta que condiciona un stress adicional al paciente, también lo condiciona a todo el equipo quirúrgico y sólo la habituación a este tipo de cirugía por parte de todo el equipo determinará unos buenos resultados ${ }^{16}$.

\section{Edema cerebral}

El edema cerebral en el momento de la cirugía se podría básicamente deber a dos causas. Primera de todas, por el uso de las estimulaciones cerebrales directas y en segundo lugar y básicamente en pacientes despiertos por una hipercapnia que condicionara una vasodilatación cerebral excesiva.

La primera causa podría deberse a un uso incorrecto de la técnica, entre ellas estimulaciones muy prolongadas, empleo de intensidades excesivamente altas... El seguimiento de la técnica y los límites presentados por Duffau ha demostrado ser seguro y no lesivo para el parénquima cerebral.

En nuestra serie no constatamos ningún caso de edema cerebral. Hecho que tampoco se constata en la larga serie descrita por Duffau.

Cuando el paciente se mantiene "despierto" no podemos descuidar que el edema cerebral puede ser consecuencia de una retención excesiva de $\mathrm{CO}_{2}$. En este caso, pasan a ser muy importantes los antecedentes del paciente (enfermedades respiratorias, síndrome de sleep apnea...), la colocación del paciente o la técnica anestésica, entre otros. Así pues, esta complicación puede ser generada por distintas razones e inevitablemente debemos reconocer el motivo para así llegar a su solución.

Según la serie de See ${ }^{16}$ registran dos casos (12\%) que fueron tratados con la administración de manitol sin afectar a la resección tumoral. La serie de Pitch $^{13}$ presentó dos casos de 20, donde uno de ellos requirió revertir a anestesia general para controlar la hiperventilación.

\section{Crisis comiciales}

En nuestra casuística las crisis comiciales sucedieron hasta en el $20 \%$. Aunque la incidencia fue alta, todos los casos se resolvieron con la aplicación de suero frío en la zona estimulada, sin requerir otras maniobras.

Por la experiencia de Duffau (en su serie de pacientes despiertos) esta complicación intraoperatoria tiene una incidencia menor del 5\% de los casos. En todos ellos no se requirió la interrupción de la cirugía. El autor Skucas ${ }^{18}$
2010; 21: 99-107

refiere una incidencia del 3\%. Muy similar es la incidencia intraoperatoria presentada por Taylor ${ }^{19}$, del $5.6 \%$, pero en este caso precisando conversión a anestesia general en un caso. En la serie de Sertelis ${ }^{17}, 17$ pacientes de $610(2.8 \%)$ dos casos requirieron la conversión a anestesia general.

La incidencia de este acontecimiento en nuestra serie respecto a la literatura fue elevada. Su aparición puede atribuirse a diferentes motivos: una mala técnica quirúrgica, un grado de infiltración tumoral determinado que sea motivo de los cambios necesarios en los parámetros de las estimulaciones, entre otros... Los casos registrados no fueron únicamente los primeros, así pues no pudimos atribuirlo a la curva de aprendizaje. Para abortar esta complicación habitualmente el uso de suero frío irrigando sobre la superficie de la corteza estimulada recientemente, es en la mayor parte de los casos suficiente (todos los casos en nuestra serie). En casos más rebeldes puede ser útil la administración endovenosa de benzodiazepinas, propofol... pudiendo interferir dificultosamente en el mapeo. Si aún así se mantienen, la conversión a una anestesia general puede ser imperativo.

Por tanto, las crisis comiciales pueden impedir la continuación del mapeo, ya fuera por déficits postcríticos, necesidad de utilización de fármacos sedantes para yugular las crisis o por generación de ansiedad al paciente.

Para un mejor manejo y evitar o minimizar este contratiempo, es útil la incorporación durante el tiempo quirúrgico del mapeo, el uso de la electrocorticografía, que en un futuro próximo queremos incorporar en nuestra práctica habitual.

Las demás complicaciones las podemos constatar selectivamente en los pacientes sometidos a una anestesia local y sedación, como son las complicaciones respiratorias, aparición de náuseas o vómitos, dolor o incomodidad postural intraoperatoria, intoxicación por anestésico local... Antes de que sobrevengan estas incidencias, no podemos olvidar que una selección cuidadosa de los candidatos a este tipo de cirugía puede hacer disminuir considerablemente la aparición de muchas de ellas. Lo mismo ocurre con la colocación del paciente o la técnica anestésica que seguiremos durante el procedimiento. Antes de profundizar con las restantes complicaciones, hemos creído oportuno considerar estas variables.

\section{Selección del paciente}

El paso básico e imprescindible para disminuir el número de complicaciones es realizar una selección ajustada para los pacientes candidatos a este tipo de cirugía. En este paso la figura del neurocirujano es esencial. Sólo él podrá indicar la existencia de un posible candidato a este tipo de cirugía que posteriormente requerirá del consenso con el resto del equipo. Es decir, el neurocirujano debe englobar todos los conocimientos anatómicos y funcionales 
para considerar indicada esta cirugía, a su vez debe valorar las condiciones clínicas en que se encuentra el paciente que puedan permitir este tipo de técnica y debe estimar si el paciente colaborará de forma adecuada durante el procedimiento.

La única contraindicación absoluta de esta técnica es un paciente no colaborador ${ }^{14}$, así como déficits neurológicos graves que pudieran distorsionar los resultados del mapeo o según algunos autores un síndrome grave de sleep apnea ${ }^{13}$. Pacientes con reflujo esofágico, pacientes muy obesos o con ansiedad extrema, tumores muy vascularizados pueden condicionar problemas potenciales intraoperatorios.

La duda de una indicación quirúrgica por un problema médico potencial deberá ser resuelta después de la discusión entre cirujano, anestesista y a veces también con el paciente ${ }^{14}$. La familiarización con esta técnica permite que el neurocirujano disponga de más habilidad para la selección de los candidatos a este tipo de cirugía.

Tampoco podemos olvidar que la motivación de los pacientes para este tipo de cirugía es imprescindible para asegurarnos su colaboración y esto conlleva tiempo. Este tiempo deberá ser invertido por todos los miembros del equipo quirúrgico antes del procedimiento para favorecer la adaptación del paciente a cada momento de la cirugía explicándole el proceso quirúrgico y su tarea.

\section{Colocación del paciente}

En nuestros primeros casos, la posición en decúbito lateral fue causa de gran incomodidad para el paciente, así que prescindimos de esta posición en los casos posteriores siempre que fuera posible técnicamente. Coincidimos al igual que $\mathrm{See}^{16}$, que la colocación de los pacientes en decúbito supino mejoraba su confort y en algunos casos, el acceso quirúrgico. También describe Whittle ${ }^{21}$ que evitan la posición en decúbito lateral para eludir los puntos de presión y tener una movilidad más cómoda del cuerpo del paciente.

Por el contrario hay autores que defienden la posición en decúbito lateral básicamente para disminuir el riesgo de obstrucción de la vía aérea ${ }^{13}$.

En algunas series desaconsejan la fijación de la cabeza del paciente para disminuir el dolor de los pins del craniostato, evitar lesiones de movimientos inesperados (ejemplo crisis epilépticas) o bien permitir una rápida intubación en caso necesario ${ }^{13}$. En nuestra serie, en todos los casos se usó el craniostato para la sujeción de la cabeza, sin comportar ningún riesgo para la seguridad del paciente.

No podemos olvidar que una vez colocado el paciente se tiene que reducir al máximo el movimiento de las personas del equipo que participan en la intervención para mantener una atmósfera lo más calmada posible ${ }^{16}$.

\section{Técnica anestésica}

El manejo anestésico en una craneotomía despierta se basa en conseguir tener al paciente suficientemente confortable como para permanecer inmóvil durante el procedimiento pero suficientemente alerta y cooperador para completar los tests neurológicos durante la cirugía ${ }^{16}$.

Inicialmente se utilizaba bolus intermitentes de fentanilo y droperidol (la conocida neuroleptoanestesia). Actualmente la combinación de propofol y reminfentanilo es la más utilizada ${ }^{16,20}$. Se ha propuesto también la utilización de la dexmetomidina, que condiciona sedación y analgesia sin interferir en el mapeo, pero todavía es escasa su experien$\operatorname{cia}^{12,20}$

La infusión de remifentanilo combinada con propofol es muy usada en la cirugía del mapeo cerebral en pacientes con ventilación espontánea. El remifentanilo tiene una vida media muy corta, la cual es independiente (no se acumula) del grado de infusión y permite un rápido control de la profundidad de la anestesia. Condiciona un mejor control de la estabilidad hemodinámica ${ }^{14}$.

Por nuestra experiencia la modalidad de infusión en TCI condiciona un mejor ajuste de la dosis administrada a cada paciente según su peso, y evita así la sobredosificación. La sobredosificación de remifentanilo que implica las complicaciones respiratorias, se asocia también a hipotensión y bradicardia $^{16}$.

\section{Intoxicación por anestésico local}

Los anestésicos locales son fármacos seguros pero tienen el potencial de generar graves problemas en caso de sobredosificación. Cuando sucede una intoxicación por anestésicos locales, la secuencia de sistemas afectados dependerá de la vía de administración y la velocidad en que ocurre los niveles tóxicos en plasma. Si los niveles en plasma aumentan lentamente, el sistema nervioso central es el primer órgano afectado. Los síntomas son generalmente excitatorios, los pacientes pueden referir parestesias periorales y de la lengua, gusto metálico, mareo, alteraciones del habla, diplopia, tinnitus, confusión, convulsiones hasta llegar al coma. Seguidamente a los síntomas neurológicos pueden aparecer los síntomas cardíacos salvo que sea por inyección intravascular que pueden aparecer inmediatamente. Puede aparecer: bradicardia, alargamiento del intervalo PR, o ensanchamiento del complejo QRS. El incremento de niveles tóxicos puede acabar desencadenando arritmias hasta llegar a la fibrilación ventricular. El manejo es el mismo que en la toxicidad del sistema nervioso central puesto que es un tratamiento de soporte (buena oxigenación, fluidos, vasopresores, inotropos, anticomiciales...). No podemos olvidar que por el efecto neuroprotector de algunos anestésicos locales las maniobras de resucitación deben ser prolongadas ${ }^{11}$. Existen casos descritos en este tipo de cirugía como son los dos casos descritos por la serie de Archer ${ }^{1}$. Aunque la incidencia es 
casi nula en otras series, debemos conocerla y evitarla. Para evadir esta complicación debemos realizar la aspiración antes de la inyección lenta, administrar las dosis de forma fraccionada, así como mantener el tiempo adecuado entre las dosis y no superar las dosis tóxicas ${ }^{11}$.

\section{Complicaciones respiratorias}

Las complicaciones respiratorias representan unas de las complicaciones más temidas en la cirugía del mapping con el paciente despierto.

Durante este tipo de cirugía el equipo quirúrgico debe estar preparado para episodios de desaturación, que pueden ser desde episodios breves y rápidamente solucionables a episodios que se pueden prolongar en apneas graves. El equipo anestésico tomará las mediadas adecuadas en cada situación, pero como neurocirujanos debemos estar advertidos que apneas serias y mantenidas pueden terminar con la intubación mecánica del paciente y condicionar el final del mapeo.

La incidencia de estas complicaciones descrita en la literatura es muy variable. Según la serie de Skucas y $\mathrm{Artru}^{18}$ de 332 casos, 5 pacientes presentaron episodios de desaturación severa $(<90 \%)(1,5 \%$ de los casos). De los cinco pacientes, tres de ellos requirieron control urgente de la vía aérea precisando intubación orotraqueal o la colocación de LMA (mascarilla laríngea). Según estos autores el IMC $>30$ es el factor de mayor riesgo de desaturación. Por el contrario, según la amplia experiencia del equipo de Duffau ${ }^{6}$, el riesgo de tener que convertir la cirugía a una anestesia general por problemas respiratorios es casi nulo.

En la serie de Mannien ${ }^{10}$, tuvieron el $18 \%$ de casos con complicaciones respiratorias ( 9 de 50 pacientes), siendo la más frecuente la depresión respiratoria. Estos eventos fueron fácilmente tratados puesto que las drogas utilizadas tienen un tiempo de vida muy corto y los pacientes pudieron ser ventilados mediante mascarilla de forma transitoria.

Actualmente se ha incrementado el uso de la mascarilla laríngea (LMA) durante la fase "paciente dormido". Con ella el paciente puede mantenerse en respiración espontánea o ventilación mecánica. Los argumentos para su uso son que la depresión respiratoria es frecuente y la LMA permite controlar la respiración, reduce la hipercapnia, mejora el confort del paciente y es de fácil colocación y extracción ${ }^{20}$.

Sea cuál sea la técnica anestésica utilizada es esencial que el equipo anestésico tenga un plan de actuación y equipo necesario en caso de tener que actuar delante de las complicaciones respiratorias ${ }^{10}$.

Frente esta complicación, para algunos autores, la colocación del paciente en decúbito lateral es una profilaxis indispensable para disminuir el riesgo de obstrucción de
2010; 21: 99-107

la vía aérea ${ }^{13}$ como anteriormente hemos mencionado. Por el contrario, algunos autores como $\mathrm{See}^{16}$ que colocan a los pacientes en decúbito supino para mejorar el confort del paciente y el acceso quirúrgico, no presentan más episodios de desaturación que otras series (18\%). Coincidimos con estos autores puesto que la colocación de los pacientes en decúbito supino con una ligera inclinación de la cabeza lateralmente, condiciona este mayor confort al paciente y facilita a su vez el acceso quirúrgico, sin tener que condicionar mayor riesgo de complicaciones respiratorias.

De nuestra serie, los episodios de desaturación que presentaron dos pacientes fueron resueltos mediante la ventilación con mascarilla sin tener que recurrir a la ventilación mecánica. Ambos pacientes tenían un IMC no superior a 30. Uno de ellos al presentar gran incomodidad postural hecho que pudo condicionar el aumento de la dosis de remifentanilo y el segundo paciente la colocación quirúrgica fue en decúbito supino.

\section{Complicaciones hemodinámicas}

Skucas ${ }^{18}$ afirmó que las complicaciones hemodinámicas eran más frecuentes en los pacientes intervenidos despiertos que bajo anestesia general. El reconocimiento de éstas y la instauración rápida de un tratamiento apropiado no comporta generalmente secuelas negativas para el paciente.

La hipertensión (PAS> 160 o PAD>90) es una complicación frecuente durante este procedimiento ${ }^{16}$. En su serie la incidencia se registra en 4 pacientes (24\%). Precisando tratamiento con labetalol endovenoso en 3 de ellos. No registra hipotensión (PAS $<90)$ ni pérdida sanguínea significativa para registrar transfusión sanguínea. No documenta casos de taquicardia $(\mathrm{FC}>120)$ o bradicardia $(\mathrm{FC}<60)$.

Se constatan dos casos de hipotensión (de 241 pacientes) intervenidos con esta técnica en la serie de Blanshard ${ }^{3}$, sin justificar su causa.

En la serie de Manninem ${ }^{10}$ registraron un caso de HTA y dos casos de hipotensión. En uno de estos últimos, la hipotensión súbita que presentó el paciente precedió a una crisis comicial que al controlarse la misma se corrigió la hipotensión.

\section{Otras complicaciones}

\section{Confusión}

Según la serie de $\mathrm{See}^{16}$, presentan un caso de confusión (6\%). Esta complicación puede ser manejada fácilmente modificando la dosis de la medicación de sedación, o a través de pequeños cambios como pueden ser modificando la temperatura ambiental, la cantidad de luz, mejorando o modificando los decúbitos del paciente ${ }^{8}$

Una preparación prequirúrgica adecuada puede ayudar a disminuir la incidencia de esta complicación. Ello supone 
dedicar un tiempo suplementario para explicar extensamente al paciente los pasos de esta cirugía y poder "asegurar" así su colaboración.

\section{Dolor e incomodidad postural}

Según la serie de $\mathrm{See}^{16}$ tres casos $(18 \%)$ requirieron la administración de analgesia adicional. El estudio de Whittle ${ }^{21}$, donde se analiza las percepciones de 15 pacientes sometidos a una intervención despiertos, detecta que el $20 \%$ de ellos refieren dolor y presumen que el lugar de fijación de los pins, así como la incisión son la mayor causa del dolor y disconfort. Ellos evitan la posición en decúbito lateral para evitar los puntos de presión y tener una movilidad más cómoda.

En nuestros primeros casos, la posición en decúbito lateral fue causa de gran incomodidad o dolor postural para el paciente. Prescindimos de esta posición en los casos posteriores siempre que fuera posible técnicamente.

Igualmente, para mejorar el confort del paciente decidimos pautar el sondaje urinario de forma habitual para evitar el deseo de micción durante la intervención quirúrgica y tener un mejor control del balance de líquidos del paciente.

\section{Náuseas y vómitos}

Según la serie de $\mathrm{See}^{16}$ presentaron un caso con náuseas que fueron controladas con Ondansetron. La serie de Blanshard ${ }^{3}$, refiere dos pacientes con episodios de vómitos intraoperatorios (de 241 casos intervenidos), sin repercusión en las vías aéreas. La serie de Manninen ${ }^{10}$ no registra ningún caso de náuseas o vómitos al igual que nuestra serie.

La manipulación quirúrgica de la duramadre, la estimulación eléctrica del lóbulo temporal e insular así como la manipulación de los vasos meníngeos puede ser la causa de estos vómitos y náuseas.

El uso de propofol (antiemético) y el menor uso de opiodes, condiciona según Manninen y $\operatorname{Tan}^{9}$ una menor incidencia de náuseas y vómitos después de este tipo de cirugía que aquéllas bajo anestesia general.

\section{Embolismo aéreo}

Se considera que la incidencia de esta complicación no es más frecuente que cualquier intervención neuroquirúrgica bajo anestesia general ${ }^{8}$. Estos autores refieren un caso de 241 pacientes $(0.04 \%)$, intervenido en posición sentado. En nuestra serie no tuvimos ningún paciente con esta complicación.

\section{Alargamiento del tiempo de la cirugía}

Se precisa de una curva de aprendizaje, no sólo desde el punto de vista quirúrgico como tal, si no también de todo el equipo quirúrgico (anestesiólogo, neurocirujano, equipo de enfermería, logopedas...). El promedio de la duración media de nuestras intervenciones es de 5 horas, pero como se ha mencionado la curva de aprendizaje de todo el equipo quirúrgico puede reducir este tiempo.

Revisando en la literatura, según la serie de Taylor ${ }^{19}$ el tiempo medio de la cirugía era de 3.25 horas. En la serie registrada por Duffau 6 , la duración media de la intervención es de 4 horas. Estos tiempos no difieren mucho más que las craneotomías habituales sin usar esta técnica.

\section{Conclusiones}

El uso de las estimulaciones cerebrales directas es seguro siempre que se realice siguiendo las indicaciones y los parámetros recomendados. Esta técnica precisa de la colaboración y de una curva de aprendizaje de todo el equipo quirúrgico. Muchas de las complicaciones intraoperatorias pueden ser evitadas mediante una selección adecuada de los candidatos a este tipo de cirugía, una buena colocación del paciente durante la intervención y un buen manejo anestésico. Las crisis y las complicaciones respiratorias pueden ser las más frecuentes y a su vez las más temidas, puesto que pueden influir en casos problemáticos en el proceso de la cirugía. El edema cerebral, complicaciones hemodinámicas, dolor e incomodidad postural, náuseas/vómitos, y confusión son otras de las complicaciones que nos podemos encontrar durante el uso de las estimulaciones cerebrales directas. El conocimiento de estas podrá facilitarnos una rápida solución sin tener que modificar habitualmente el planteamiento inicial de la intervención.

\section{Bibliografía}

1. Archer, D.P., Jocelyne, M.A.: Conscious-sedation analgesia during craniotomy for intractable epilepsy: a review of 354 consecutive cases. Can J Anaesth 1988; 35: 338-344.

2. Berger, M., Keles, E.: Tumors in eloquent areas. En: Sekhar L, Fessler R.: Atlas of neurosurgical techniques (brain). New York; Thieme Medical, 2006.pp. 477-484.

3. Blanshard, H., Chung, F., Manninem, P., Taylor, M., Bernstein, M.: Awake craniotomy for removal of intracranial tumor: considerations for early discharge. Anesth Analg 2001; 92: 89-94.

4. Duffau, H., Capelle, L., Denvil, D., Sichez, N., Gatignol, P., Taillandier, L., et al.: Usefulness of intraoperative electrical subcortical mapping during surgery for low-grade gliomas located within eloquent brain regions: functional results in a consecutive series of 103 patients. J Neurosurg 2003; 98: 764778.

5. Duffau, H.: Peroperative functional mapping using direct electrical stimulations. Methodological considerations. Neurochirurgie 2004; 50: 474-83. 
6. Duffau, H., Lopes, M., Arthuis, F., Bitar, A., Sichez, J.P., Van Effenterre, R., et al.: Contribution of intraoperative electrical stimulations in surgery of low grade gliomas: a comparative study between two series without (1985-96) and with (1996-2003) functional mapping in the same institution. J Neurol Neurosurg Psychiatry 2005; 76: 845-851.

7. Duffau, H.: Contribution of cortical and subcortical electrostimulation in brain glioma surgery: methodological and functional considerations. Clinical Neurophysiology 2007; 37: 373-382.

8. Erickson, K., Cole, D.: Anesthetic considerations for awake craniotomy for epilepsy. Anesthesiology Clin 2007; 25: 535-555.

9. Manninen, P.H., Tan, T.K.: Postoperative nausea and vomiting after craniotomy for tumor surgery: A comparison between awake craniotomy and general anestesia. J Clin Anesth 2002; 14: 279-283.

10. Manninen, P.H., Balki, M., Lukitto, K., Bernstein, M.: Patient satisfaction with awake craniotomy for tumor surgery: a comparison of remifentanil and fentanyl in conjuntion with propofol. Anest Analg 2006; 102: 237-242.

11. McLure, H.A., Rubin, A.: Review of local anaesthetic agents. Minerva anestesiol 2005; 71: 69-74.

12. Moore, T.A., Markert, J., Knowlton, R.C.: Dexmedetomidine as rescue drug during awake craniotomy for cortical motor mapping and tumor resection. Anesth Analg 2006; 102: 1556-1558.

13. Picht, T., Kombos, T., Gramm, H.J., Brock, M., Suess, O.: Multimodal protocol for awake craniotomy in language cortex tumour surgery. Acta Neurochir (Wien) 2006; 148 : 127-138.

14. Sarang, A., Dinsmore, J.: Anasthesia for awake cranio- tomy- evolution of a technique that facilitates awake neurological testing. Br J Anasth 2003; 90: 161-165.

15. Sartorius, C., Wright, G.: Intraoperative brain mapping in a community setting- technical considerations. Surg Neurol 1997; 47: 380-388.

16. See, J.J., Lew, T., Kwek, T.K., Chin, K.J., Wong, M., Liew, Q.Y., et al.: Anasthetic management of awake craniotomy for tumour resection. Ann Acad Med Singapore 2007; 36: 319-325.

17. Serletis, D., Bernstein, M.: Prospective study of awake craniotomy used routinely and nonselectively for supratentorial tumors. J Neurosurg 2007;107: 1-6.

18. Skucas, A.P., Artru, A.A.: Anesthestic complications of awake craniotomies for epilepsy surgery. Anesthe Analg 2006; 102: 882-887.

19. Taylor, M.D., Bernstein, M.: Awake craniotomy with brain mapping as the routine surgical approach to treating patients with supratentorial intraaxial tumors: a prospective trial of 200 cases. J Neurosurg 1999; 90: 35-41.

20. Van de Wiele, B.: Anesthesia and functional brain mapping. ASA Annual Meeting 2007. San Francisco 2007: 514 (1-6) (Abstract).

21. Whittle, I.R., Midgley, S., Georges, H., Pringle, A.M., Taylor, R.: Patient perceptions of awake brain tumour surgery. Acta Neurochir (Wien) 2005; 147: 275-277.

Teixidor, P.; García, R.; Alamar, M.; González, M.; Llasera, R.; Durá, M.J.; Muñoz, J.; Florensa, R.: Complicaciones intraoperatorias del mapeo corticosubcortical. Neurocirugía 2010; 21: 99-107.

Correspondencia: pilarteixidor.germanstrias@gencat.cat 